\title{
FOURIER-STIELTJES COEFFICIENTS AND CONTINUATION OF FUNCTIONS
}

\author{
R. KAUFMAN
}

1. To introduce our subject we recall two classical problems of continuation for certain functions of a complex variable.

(CA) Here $f$ is continuous in $R^{2}$ and analytic outside a closed set $E$; $E$ is removable for this problem if it is always true that $f$ is entire.

(QC) In this problem $f$ is a homeomorphism of the extended plane, which is $K$-quasiconformal outside $E ; E$ is removable if $f$ is always quasiconformal in the extended plane.

For both problems there is a best-possible theorem. $E$ is removable if it is of $\sigma$ finite length (Besicovitch [2]; Gehring [5]). In each problem, a product set $F \times[0,1]$ is non-removable precisely when $F$ is uncountable (Carleson [3], Gehring [5]). Our purpose is to find nonremovable sets contained in $F \times[0,1]$, not of the product type nor even approximately so. To describe these sets we denote by $\Gamma$ a compact set in $R^{2}$ meeting each line $x=x_{0}$ at most once, so that $\Gamma$ is the graph of a real function whose domain is a compact set in $R$; since $\Gamma$ is closed, that function is continuous.

Theorem. (a) In each compact set $E_{1} \times E_{2}$, where $E_{1}$ is uncountable and $E_{2}$ has positive linear measure, there is a graph $\Gamma$ non-removable for (CA).

(b) In each set $E_{1} \times[0,1]$, where $E_{1}$ is uncountable, there is a graph $\Gamma$, non-removable for (QC).

The reason for requiring an interval on the $y$-axis, and not merely a set of posi tive measure in (b), can be seen from [1, p. 128]. Perhaps the correct class of sets $E^{2}$ could be found. When $E_{2}$ is an interval, the non-removability of $\Gamma$ can be improved in two directions.

2. Both proofs are based on a theorem of Wiener (1924) about the FourierStieltjes transforms of measures on $R$; a streamlined version of this theorem is presented in $\left[6 ;\right.$ p. 42]. We adopt the symbol $e(t) \equiv e^{2 \pi \text { it }}$ and the notation $\hat{\mu}(u) \equiv \int e(-u t)$. $\mu(d t)$. Wiener's theorem is then the relation $\lim _{N}(2 N+1)^{-1} \sum_{-N}^{N} \hat{\mu}(k)=\mu(Z)$, and in fact is an easy consequence of dominated convergence. When $\mu$ is continuous, i.e. has no jumps, and $\lambda=\mu * \tilde{\mu}$ is defined by $\hat{\lambda}=|\hat{\mu}|^{2}$, this becomes $\sum_{-N}^{N}|\hat{\mu}(k)|^{2}=o(N)$. This means that there is a set $N_{1}$ of positive integers, of asymptotic density 1 , such 
that $\hat{\mu}(k) \rightarrow 0$ as $k \rightarrow \infty$ in $N_{1}$. The Lebesgue space $L^{1}(d \mu)$ is separable so there is a set $N_{2}$, again of asymptotic density 1 , such that $e(-k t) \rightarrow 0$ weak* in $L^{\infty}(d \mu)$ as $k \rightarrow \infty$ in $N_{2}$, that is $\int e(-k t) f(t) \mu(d t) \rightarrow 0$ for each $f$ in $L^{1}(d \mu)$. One more use of the same device yields the following variant of Wiener's theorem for continuous measures $\mu$ in $R$ :

(W) There is a sequence $1 \leqq q_{1} \leqq q_{2} \leqq \ldots \leqq q_{v} \leqq \ldots$ such that $e\left(-p q_{v} t\right) \rightarrow 0$ weak w $^{*}$ in $L^{\infty}(d \mu)$ as $v \rightarrow \infty$, for each $p= \pm 1, \pm 2, \pm 3, \ldots$. (Plainly we could add the condition $q_{v}=v+o(v)$, but asymptotic density 1 is used merely to find the sequence and has no further use.)

Following [3] and [5], we fix a continuous probability measure $\mu$ in $E_{1}$.

3. Proof of (a). Let now $E_{2}$ be a compact set of positive linear measure on the $y$ axis; there exists a function $\varphi(z)$, analytic off $E_{2}$, such that $\varphi=z^{-1}+\ldots$ near $\infty$ and $|\varphi| \leqq C_{1}$ on $R^{2} \backslash E_{2}$. (The constant $C_{1}=4 / m\left(E_{2}\right)$ was found by Pommerenke [8] and is always the minimum value; see also [4; pp. 28-30]. The value of $C_{1}$ has no significance in the sequel.)

By Fatou's theorem for the half-plane, $\varphi$ admits one-sided limits a.e. on $E_{2}$; using Cauchy's formula and taking limits we obtain $\varphi(\zeta)=\int g(y)(i y-\zeta)^{-1} d y$, for all $\zeta \notin E_{2}$, where $g \in L^{\infty}, g=0$ off $E_{2}$.

Let now $H \in L^{\infty}(R) \cap C^{1}(R)$ and $\psi(\zeta)=\int g(y) H(y)(i y-\zeta)^{-1} d y, \quad \zeta \notin E_{2}$. Then with $\zeta=\xi+i \eta$, we can write

$$
\psi(\zeta)-H(\eta) \varphi(\zeta)=\int[H(\eta)-H(\zeta)] g(y)(i y-\zeta)^{-1} d y .
$$

The last formula shows plainly that $\psi$ can be estimated by means of $\|\varphi\|_{\infty},\|H\|_{\infty}$, $\left\|H^{\prime}\right\|_{\infty}$, and the measure of $E_{2}$.

4. Proof of (a), completed. Suppose now that the function $g$, the measure $\mu$, the sequence $\left(q_{v}\right)$, and an integer $p \neq 0$ are held fixed. We form the sequence of functions

$$
\psi_{v}(\zeta)=\int e\left(-p q_{v} x\right) \int e(p y) g(y)(x+i y-\zeta)^{-1} d y \mu(d x) .
$$

For fixed $x \in E_{1}$, the inner integral is defined whenever $\xi \neq x$, and is $O(p)$, or indeed $O(1+\log |p|)$, hence $\psi_{v}(\zeta)$ is defined for all $\zeta$, and is continuous in $R^{2}$, by the continuity of $\mu$ and dominated convergence. We claim that $\psi_{v} \rightarrow 0$ uniformly as $v \rightarrow+\infty$. To verify this claim we consider the integrals $\int e(p y) g(y)(x+i y-\zeta)^{-1} d y$ as elements of $L^{1}(d \mu)$, parametrized by a complex number $\zeta$. Dominated convergence shows that this collection of functions is norm-compact in $L^{1}(d \mu)$; since $e\left(-p q_{v} x\right) \rightarrow 0$ weak* in $L^{\infty}(d \mu)$ the uniform convergence follows.

It is now a simple matter to complete the proof. We suppose that $E_{2}$ has diameter $<1 / 2$, as we can without loss of generality. Beginning with

$$
f_{0}(\zeta)=\int \varphi(\zeta-x) \mu(d x)=\iint g(y)(x+i y-\zeta)^{-1} d y \mu(d x)
$$


we replace $f_{0}$ by an integral

$$
f_{1}(\zeta)=\iint H_{1}\left(y-q_{v} x\right) g(y)(x+i y-\zeta)^{-1} d y \mu(d x)
$$

where $H_{1} \in C^{2}(R), H_{1}$ has period 1 and mean 1 , and $H_{1}(t)=0$ outside the set $|t| \leqq$ $1 / 4$ (modulo 1). Hence

$$
H_{1}\left(y-q_{v} x\right)=1+\sum^{\prime} a_{p} e\left(p y-p q_{v} \cdot x\right)
$$

where $\sum\left|p a_{p}\right|<+\infty$. Thus

$$
f_{1}(\zeta)-f_{0}(\zeta)=\Sigma^{\prime} a_{p} \int e\left(-p q_{v} x\right) \int e(p y) g(y)(x+i y-\zeta)^{-1} d y \mu(d x) .
$$

The previous analysis establishes that $f_{1}$ is continuous in the plane, and that $f_{1} \rightarrow f_{0}$ uniformly as $v \rightarrow \infty$. Moreover $f_{1}$ is analytic off the set defined by $x \in E_{1}, y \in E_{2}$ and the relation $\left|y-q_{v} x\right| \leqq 1 / 4$ (modulo 1). Since $E_{2}$ has diamter $<1 / 2$, each line $x=x_{0}$ meets the set of singularities in a set of diameter $<1 / 2$. We choose $v=v_{1}$ so that $\left|f_{1}-f_{0}\right|<1 / 2$, say, and then construct $f_{2}$ by inserting a factor $H_{2}\left(y-q_{v_{2}} x\right)$, etc. $H_{2}(t)=0$ outside the set $|t|<1 / 8$ (modulo 1 ), etc. The limit $f$ is then continuous in $R^{2}$ and analytic off the support of each function $H_{k}\left(y-q_{v_{k}} x\right)$ and off $E_{1} \times E_{2}$. Hence the (closed) set of singularities is already a graph $\Gamma \subseteq E_{1} \times E_{2}$. To ensure that $f$ is not entire we have only to control the Taylor expansion of $f_{1}, f_{2}, \ldots$ at $\infty$. Now $f_{0}(\zeta)=\zeta^{-1}+\ldots$, and the functions $f_{k}$ are analytic outside a fixed compact set; hence the first coefficient at $\infty$ can be controlled simply by writing it as an integral around a large circle. (If $f$ were entire, it would be constant.)

As mentioned before, a better result is possible when $E_{2}$ is an interval: all the derivatives $f^{\prime}, f^{\prime \prime}, \ldots$ are uniformly continuous off $\Gamma$. To see this we choose $g(y)$ to be $\mathrm{sm}_{\mathrm{O}}$ oth, as well as $H_{1}, H_{2}, \ldots$, and estimate the partial derivatives $\partial / \partial y, \partial^{2} / \partial y^{2}, \ldots$ at each step, using Leibniz' formula. We observe that if $E_{1}$ and $E_{2}$ have no interior, if $f$ is analytic off $E_{1} \times E_{2}$, and $f^{\prime}$ is uniformly continuous there, then $f$ is entire. (T hus the improvement just mentioned is not possible if $E_{2}$ has no interior; if $E_{1}$ has measure 0 and $E_{2}$ has no interior, then $f^{\prime}$ cannot even remain bounded unless $f$ is entire.)

5. Proof of (b). Let $g(y)=\sin ^{2} 2 \pi y, 0 \leqq y \leqq 1 / 2$ and $g(y)=0$ otherwise. We construct a sequence of real-valued functions

$$
\begin{gathered}
u(x, y)=\int_{-\infty}^{x} g(y) \mu(d y) \equiv g(y) \mu(-\infty, x) \\
u_{k}(x, y)=\int_{-\infty}^{x} A_{1}(t, y) \ldots A_{k}(t, y) g(y) \mu(d t),
\end{gathered}
$$

where each $A_{k} \geqq 0$ and $A_{k} \in C^{2}(R)$. Then of course $u_{k}(x, y)$ is continuous and increasing for each fixed $y, \partial u_{k}(x, y) / \partial y$ exists everywhere as a classical derivative and $\partial u_{k} / \partial x=0$ away from the support of the measure $A_{1}(x, y) \ldots A_{k}(x, y) \mu(d x)$. Also, $A_{k}(x, y)=H_{k}\left(y-q_{v} x\right)$ where $v=v_{k}$ is chosen as follows. Abbreviating $G_{k}=A_{1} \ldots A_{k}$ 
we have

$$
\begin{gathered}
u_{k+1}(x, y)=\int_{-\infty}^{x} H_{k+1}\left(y-q_{v} t\right) G_{k}(t, y) g(y) \mu(d t), \\
\partial\left(u_{k+1}-u_{k}\right) / \partial y=\int_{-\infty}^{x} H_{k+1}^{\prime}\left(y-q_{v} t\right) G_{k}(t, y) g(y) \mu(d t) \\
+\int_{-\infty}^{x}\left[H_{k+1}\left(y-q_{v} t\right)-1\right]\left(\partial\left[G_{k}(t, y) g(y)\right] / \partial y\right) \mu(d t) .
\end{gathered}
$$

Since $G_{k}$ and $g(y)$ are at least $C^{1}$, the following estimation of the first term here will also be valid for the second one. We expand $H_{k+1}^{\prime}$ in a Fourier series; observing that the constant term $(p=0)$ is now absent. Hence everything is reduced to estimation of the integrals

$$
2 \pi i p \int_{-\infty}^{x} G_{k}(t, y) e\left(p y-p q_{v} t\right) g(y) \mu(d t)
$$

which are clearly $O(p)$. To proceed as in (a), we need a norm-compact subset in $L^{1}(d \mu)$; we define it as the set of all functions $G_{k}(t, y) I(t \leqq x)$ with $x+i y$ in $R^{2}$, $I$ means characteristic function. With these adaptations, we obtain the uniform convergence of $u_{k+1}$ and $\partial u_{k+1} / \partial y$. A further property of $u_{k}$ is necessary and easily obtained: $u_{k}(+\infty, y)-u_{k}(-\infty, y) \geqq c>0$ when $1 / 8 \leqq y \leqq 3 / 8$, with $c$ independent of $k$; obviously $u_{0}$ has this property. We carry out an infinite sequence of approximations, observing that $\left|\partial u_{0} / \partial y\right| \leqq 2 \pi<7$, obtaining a real function $u$ such that $|\partial u / \partial y|<7$ everywhere and $\partial u / \partial x=0$ away from $\Gamma$, for a certain graph $\Gamma \subseteq E_{1} \times[0,1]$. Now $f(x, y)=u(x, y)+x+i y$ is a homeomorphism of $R^{2}$ onto itself with $f(\infty)=\infty, f$ is of class $C^{1}$ off $\Gamma$ and $f$ is $K$-quasiconformal off $E$. We verify the latter point following a suggestion of the referee: off $\Gamma$ we have $\left|f_{x}\right|^{2}+\left|f_{y}\right|^{2}=1+u_{y}^{2}+1 \leqq 51$ while the determinant $J=u_{x}+1=1$. However $f$ cannot be quasiconformal in the plane, because $f(\Gamma)$ has area at least $c / 4$, as seen from the properties of $u$ on horizontal lines.

By Lemma 3.1 of $[8$, p. 200], the curve $\Gamma$ is not removable even when $K=1$, i.e. when the homeomorphism is complex analytic off $\Gamma$; this means that it is in general not even $K$-quasiconformal in the plane for any $K<+\infty$.

(In a subsequent note on exceptional sets we present a method for constructing conformal mappings that avoids the Beltrami equation but uses more algebra.)

6. The sets $E_{1} \times E_{2}$ are not quite the most general that can be handled by Carleson's method and through which a curve $\Gamma$ can be passed.

Suppose that $E \subset R^{2}$ is compact and the set $\{x \in R: m(E(x))>0\}$ is uncountable. (Here $E(x)$ is the section of $E$ through $x$.) Each set $\{m(E(x)) \geqq c\}$ is closed, so that for some $c>0$ that set carries a continuous probability measure $\mu$. Let $h_{n}(x, y)$ be continuous in $R^{2}, 0 \leqq h_{n} \leqq 1$, and $\lim h_{n}=\chi_{E}$ everywhere. Supposing that $\iint\left|h_{n}(x, y)-\chi_{E}(x, y)\right| \mu(d x) d y<n^{-2}$, we can find a set $B \subseteq R$ of positive $\mu$-measure, such that $\int\left|h_{n}(x, y)-\chi_{E}(x, y)\right| d y \rightarrow 0$ uniformly for $x \in B$ as $n \rightarrow+\infty$. To each section $E(x), x \in B$, there is a function $\varphi$, analytic off $E(x)$ and bounded by some $c^{\prime}$, while $\varphi(\zeta)=\zeta^{-1}+\ldots$ near $\infty$. This can be obtained by an explicit construction, show- 
ing that $\varphi$ depends continuously on $x$ in an appropriate topology. This is a perfect substitute for the compactness used before and so our proof for (CA) can be effected.

Supported by the National Science Foundation and the Center for Advanced Study (Urbana).

\section{References}

[1] Ahlfors, L., and A. Beurling: Conformal invariants and function-theoretic null-sets. - Acta Math. 83, 1950, 101-129.

[2] Besicovitch, A.: On sufficient conditions for a function to be analytic, and on behaviour of analytic functions in the neighbourhood of non-isolated singular points. - Proc. London Math. Soc. (2) 32, 1931, 1-9.

[3] Carleson, L.: On null-sets for continuous analytic functions. - Ark. Mat. 1, 1950, 311-318.

[4] Garnett, J.: Analytic capacity and measure. - Lecture Notes in Mathematics 297, SpringerVerlag, Berlin-Heidelberg-New York, 1972.

[5] Gehring, F. W.: The definitions and exceptional sets for quasiconformal mappings. - Ann. Acad. Sci. Fenn. Ser. A I Math. 281, 1960, 1-28.

[6] Katznelson, Y.: An introduction to harmonic analysis. - John Wiley \& Sons, Inc., New YorkLondon-Sydney-Toronto, 1968. (Dover reprint, 1976.)

[7] Lehto, O., and K. I. Virtanen: Quasiconformal mappings in the plane. - Springer-Verlag, Berlin-Heidelberg-New York, 1973.

[8] Pommerenke, Chr:. Über die analytische Kapazität. - Arch. Mat. (Basel) 11, 1960, 270-277.

University of Illinois at Urbana-Champaign

Department of Mathematics

Urbana, Illinois 61801

USA

Received 14 September 1983 